\title{
Reduced order modelling applied to augmented reality
}

\author{
Alberto Badías ${ }^{1}$, Iciar Alfaro ${ }^{1}$, David González ${ }^{1}$, Elías Cueto ${ }^{1}$ \\ ${ }^{1}$ Applied Mechanics and Bioengineering (AMB) \\ Instituto de Investigación en Ingeniería de Aragón (I3A) \\ Universidad de Zaragoza, Mariano Esquillor s/n, 50018, Zaragoza, Spain. \\ Tel. +34-976762707, e-mail: abadias @ unizar.es
}

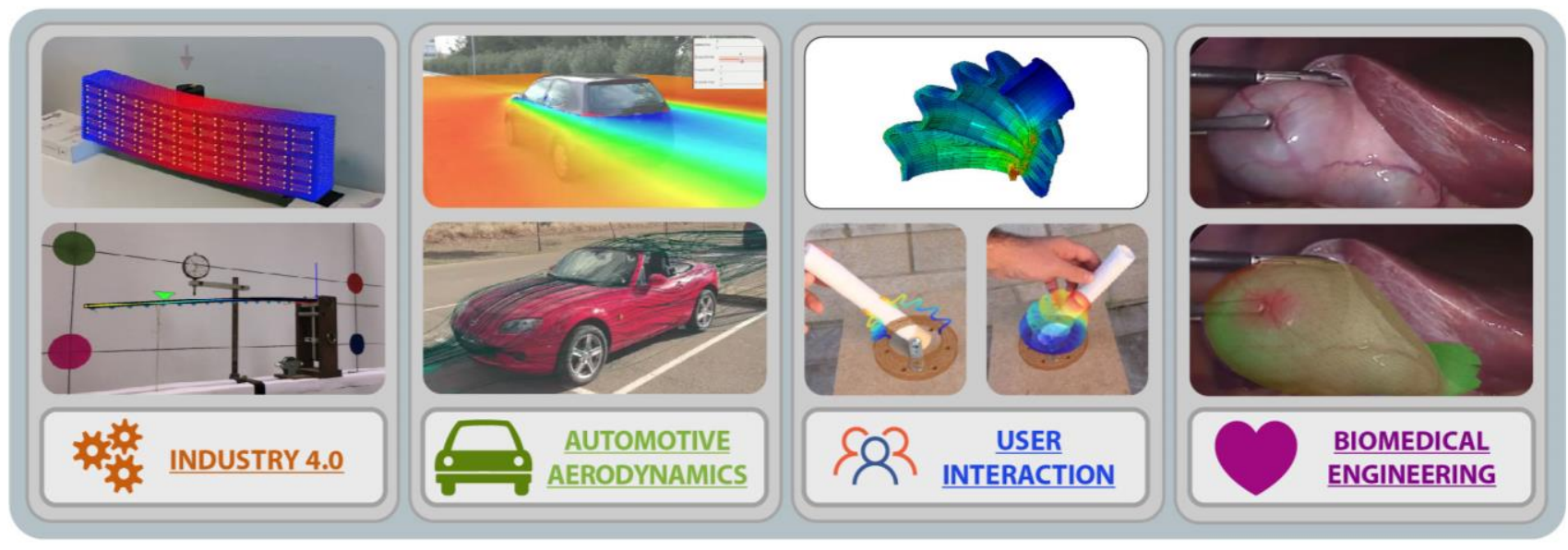

Fig. 1: Examples of our developed work, applied to multiple fields. For more info and videos, please visit the website http://amb.unizar.es/people/alberto-badias/

\begin{abstract}
Augmented reality is one of the fields with greatest interest in technological research. Real time requirements force to use physics engines to approximate the behaviour of the objects. We propose the computation of the proper equations that govern the physics of deformable objects, and their interaction with users in real time, using dimensionality reduction techniques.
\end{abstract}

\section{Methods}

In a world where technological advances surprise us every day, we still do not have the right tools to interact properly with a virtual world. Although there is no doubt it will change in a few years. Augmented reality is included inside the RealityVirtuality Continuum, being an homogeneous fusion of the real and virtual worlds. Thus, augmented reality is the addition of virtual information within our real reality (see Fig. 2).

The interaction of a user with the objects coming from a virtual world makes use, fundamentally, of techniques that belong to two large science communities: computer vision and physical simulation.

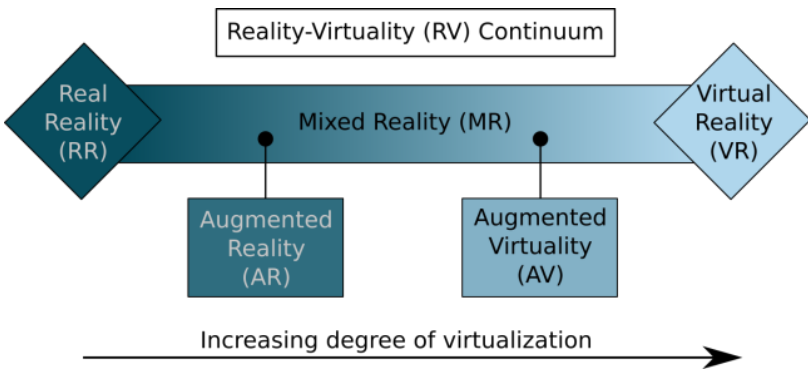

Fig. 2: Reality-virtuality continuum defined by [1] showing the real reality, the virtual reality and the mixed reality that combines information about both worlds.

\section{Computer vision}

It is necessary to make a perfect adjustment of both worlds. In other words, we have to allow the movement through the virtual world as we do in our real world. Only in this way we can perceive interactions in a natural way. We use SLAM (simultaneous localization and mapping) techniques [2] that allow us to capture the real scene around us using a monocular camera (like the camera we have in our smartphone). It is necessary to make an adjustment (registration) between both worlds in case the SLAM algorithm has a random initialization, which is usually the most common procedure. 


\section{Physical simulation}

On the other side we have the behavior of the virtual objects we want to interact with. Realistic behaviour requires physical simulations, demanding a very high computational cost which cannot be performed at video frequency. That is why we use model order reduction techniques to reduce the complexity of the computations. The aim is to carry out the simulations in two stages: a first phase with a high computational cost where the physical problem is solved in a parameterical way, and a second phase where the solution is evaluated in real time. These physical problems can be, for example, the deformable continuum mechanics [3], fluid mechanics [4] or also electromagnetism interactions [5], among others. And of course, some of them involve highly non-linear behaviour laws, which means very high computational costs.

The pre-computed parametric solution is stored in a compressed form to reduce the cost of storage in memory, but allowing negligible decompression time for the solution (building the solution in the original space). The process of compression is computed as the projection of the original solution into a subspace of reduced dimensionality. To do this, solution $\boldsymbol{u}$ of our system is approached as the sum of $N$ functions $F_{i}$ with separate dependency on $D$ parameters (Eq. 1).

$\mathbf{u}\left(x_{1}, x_{2}, \ldots, x_{D}\right) \approx \sum_{i=1}^{N} F_{i}^{1}\left(x_{1}\right) \cdot F_{i}^{2}\left(x_{2}\right) \cdot \ldots \cdot F_{i}^{D}\left(x_{D}\right)$

The aim is to solve an inverse problem in order to estimate the parameters of the simulation in real time. Talking about object deformations (continuum mechanics), this is done by solving the following functional

$$
\mathscr{J}(\mu)=\sum_{j=1}^{n_{\text {meas }}}\left(\mathbf{u}_{\text {pix }}^{\text {meas }}\left(\mathbf{x}_{j}\right)-\Pi_{t}\left(\mathbf{u}^{\text {MOR }}\left(\mathbf{x}_{j}, \mu\right)\right)\right)^{2}
$$

being $n_{\text {meas }}$ the number of measurements, $\boldsymbol{u}_{\text {pix }}^{\text {meas }}$ the pixel coordinates measured in the image, $u^{M O R}$ the 3D displacements predicted by the reducedorder parametric solution and $\Pi_{t}$ the projection to the image plane at any instant $t$. This functional is minimized by using the Levenberg-Marquardt (LM) algorithm [6], where the expression of the reduced solution in a separate variables form provides a critical advantage, since the Jacobian in LM is

$$
\mathbf{J}_{k}=\frac{\partial \mathbf{u}(\mathbf{x}, \mu)}{\partial \mu_{k}}=
$$

$=\sum_{i=1}^{N} \mathbf{F}_{i}^{1}\left(\mu_{1}\right) \cdot \ldots \cdot \frac{\partial \mathbf{F}_{i}^{k}\left(\mu_{k}\right)}{\mu_{k}} \cdot \ldots \cdot \mathbf{F}_{i}^{n_{\text {param }}}\left(\mu_{n_{\text {param }}}\right)$

being $\boldsymbol{J}_{\boldsymbol{k}}$ the jacobian component of parameter $k, \boldsymbol{\mu}$ the set of parameters and $\boldsymbol{F}_{k}$ the separated functions depending on any parameter $k$. Since the solution is expressed in a separate variables fashion, those derivative terms of the solution for each parameter (sensitivity) are computed very fast.

\section{Conclusions}

The fusion of techniques from both scientific communities is a step forward giving very precise results, that could not be achieved separately. We use the video sequences as data input to our physical models, and the results of our simulations feedback the video sequence. This is a constant flow of information between the images and the physical models, obtaining impressive results, as can be seen in the examples of Fig. 1. The applications are very diverse, including advances in the way data is displayed in the revolutionary Industry 4.0, with real-time simulations. Of course, it can be applied also in the direct interaction with users, thinking about entertainment industry with applications in social markets. We are also developing biomedical applications in the surgery environment to add extra information to the surgeon in real-time.

\section{REFERENCES}

[1]. MILGRAM, P. and KISHINO, F. A taxonomy of mixed reality visual displays. IEICE TRANSACTIONS on Information and Systems, 1994, 77(12), pp.1321-1329.

[2]. MUR-ARTAL, R., MONTIEL, J. M. M. and TARDOS, J. D. ORB-SLAM: A Versatile and Accurate Monocular SLAM System. IEEE Transactions on Robotics, 2015, 31(5),1147-1163.

[3]. NIROOMANDI, S., et al. 1. Real-Time Deformable Models of Non-Linear Tissues by Model Reduction Techniques. Computer methods and programs in biomedicine, 2008, 91(3),223-231.

[4]. DIEZ, P., ZLOTNIK,S. HUERTA,A. Generalized Parametric Solutions in Stokes Flow. Computer Methods in Applied Mechanics and Engineering, 2017, 326223-240.

[5]. CHINESTA, F., et al. Model Reduction \& Manifold learning-Based Parametric Computational Electromagnetism: Fundamentals \& Applications. Electromagnetic Field Computation (CEFC), 2016 IEEE Conference On. IEEE, 2016, p.1.

[6]. LEVENBERG, K. A Method for the Solution of Certain Non-Linear Problems in Least Squares. Quarterly of Applied Mathematics, 1944, 2(2), 164168. 